\title{
Superconducting proximity effect in clean ferromagnetic layers
}

\author{
M. Zareyan, ${ }^{1}$ W. Belzig, ${ }^{2}$ and Yu. V. Nazarov ${ }^{3}$ \\ ${ }^{1}$ Institute for Advanced Studies in Basic Sciences, 45195-159, Zanjan, Iran \\ ${ }^{2}$ Department of Physics and Astronomy, University of Basel, Klingelbergstr. 82, 8056 Basel, Switzerland \\ ${ }^{3}$ Department of Applied Physics and Delft Institute of Microelectronics and Submicrontechnology, Delft University of Technology, \\ Lorentzweg 1, 2628 CJ Delft, The Netherlands
}

(Received 3 July 2001; revised manuscript received 4 February 2002; published 16 April 2002)

\begin{abstract}
We investigate the superconducting proximity effect in clean ferromagnetic layers with rough boundaries. The subgap density of states is formed by Andreev bound states at energies which depend on trajectory length and the ferromagnetic exchange field. At energies above the gap, the spectrum is governed by resonant scattering states. The resulting density of states, measurable by tunneling spectroscopy, exhibits a rich structure, which allows us to connect the theoretical parameters from experiments.
\end{abstract}

DOI: 10.1103/PhysRevB.65.184505

PACS number(s): 74.50. $+\mathrm{r}, 74.80 .-\mathrm{g}, 75.70 .-\mathrm{i}$

\section{INTRODUCTION}

Investigating of superconducting proximity effect in normal systems has a long history back to the experiments of McMillan and Rowell. ${ }^{1}$ Their tunneling spectroscopy measurements in normal metals connected to a superconductor revealed strong modifications of the density of states (DOS) caused by the induced superconducting correlations. These results were understood in the tunneling model of McMillan. ${ }^{2}$ He noted that the changes in the DOS of the normal metal occur on a scale $E_{\mathrm{Th}}$, which in his model is identified with inverse escape time of a quasiparticle in the normal metal. We can understand this observation by noting that in clean normal metal films the electronic properties are determined by so-called Andreev bound states. ${ }^{3}$ These are bound electron-hole pairs residing on trajectories, which start and end at the superconductor. The characteristic energy scale in this case is $v_{\mathrm{F}} / d$, again the inverse escape time. In recent years experiments became possible in which the density of states was resolved locally on a sub- $\mu \mathrm{m}$ scale. For example, the dependence of the tunneling DOS on the distance from the superconductor in normal metals has been measured by Guéron et al. ${ }^{4}$ using additional tunnel junctions. These results have been successfully explained within the quasiclassical theory in the diffusive limit. ${ }^{5}$ Other experiments made use of low-temperature scanning tunneling microscopes to resolve spatially the DOS of small droplets of normal metal on the surface of a superconductor. ${ }^{6}$ Nowadays these types of experiments are becoming a standard technique. $^{7-9}$

The question of the proximity effect in the presence of a spin splitting is currently heavily investigated. In particular the influence of a superconductor on transport properties of a ferromagnet is under debate, ${ }^{10-12}$ in which case the proximity effect is negligible. It is, however, natural to address the question of the influence of an exchange field on the proximity density of states. In fact, this question was already addressed experimentally a while ago by Gallagher et al. ${ }^{13}$ They observed a spin splitting of the DOS in thin normal layers in a parallel magnetic field. New experimental developments, ${ }^{14}$ exploring the proximity effect on a nanometer scale, demand new theoretical models, beyond the simple tunneling model of McMillan. In the present paper we investigate a new model, suitable for these experiments.

The motivation stems not only from fundamental question of the coexistence of ferromagnetism and superconductivity, but also because interesting applications of ferromagnetsuperconductor (FS) hybrid structures have been proposed. We only mention here the potential use of SFS contacts in the construction of quantum computers. SFS junctions are candidates for all-electronic $\pi$ junctions, which are needed in some proposals for solid-state qubits. ${ }^{15,16}$

Larkin and Ovchinnikov ${ }^{17}$ and Fulde and Ferrel ${ }^{18}$ (LOFF) independently predicted that in the presence of an exchange field $h$ (for instance, in magnetic superconductors) a specific superconducting state can be formed in which the order parameter has an oscillatory spatial modulation. The spatially oscillating order parameter contains nodes in which the phase changes by $\pi$. The LOFF state has never been observed in bulk superconductors, but there is recently evidence for detecting an induced LOFF state in heterostructures of ferromagnets and superconductors. Many works have investigated the thermodynamics properties of FS multilayers. Radovic et al. ${ }^{19}$ have predicted oscillations of the superconducting critical temperature $T_{c}$ as a function of the thickness of the attached $F$ layer. The experimental evidence for these $T_{c}$ oscillations is not, however, conclusive. ${ }^{20}$ The reason for this may, for example, result from the bad quality of the FS interface. ${ }^{21}$

The most recent experiments have concentrated on other properties of FS layers. Ryasanov et al. ${ }^{22}$ measured the temperature dependence of the critical current in SFS Josephson junctions with thin $\mathrm{F}$ layers and have found a nonmonotonic temperature dependence. This behavior can be understood in terms of a $\pi$ phase shift due to the exchange field, which occurs for certain values of the thickness of the F layer, as was predicted by Bulaevskii et al. ${ }^{23}$ An indirect proof of the $\pi$ phase shift has been made by Kontos et al. ${ }^{14}$ who studied the density of states in thin ferromagnetic films contacted by a superconductor. They observed an oscillatory behavior of the induced superconducting correlation for layers of different thickness, which was attributed to the influence of the exchange field. In Ref. 24, we have shown that these experimental findings could be explained by a model of a ballistic 


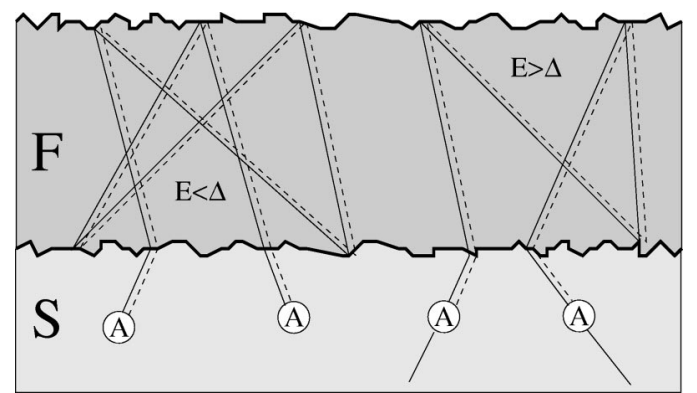

FIG. 1. Schematic drawing of our model of a ferromagnetic film (F) topping a superconductor (S). Typical classical trajectories are also indicated. We distinguish two processes. At energies $E$ below the superconducting gap $\Delta$, quasiparticles are confined to the film by Andreev reflection (indicated by the white circles). An examples for this process is given by the left trajectory. For $E>\Delta$ Andreev reflection is incomplete and the quasiparticles states in $\mathrm{F}$ are formed by scattering resonances, symbolized in the right process.

ferromagnetic layer with rough boundaries. The best agreement was obtained in the limit of large $h / \Delta$ and small interface transparency $T$.

In the present paper, we study the proximity DOS in a clean ferromagnetic layer on top of a superconductor in the full parameter range. Within the ballistic quasiclassical formalism we obtain that the DOS for all energies is completely specified by the length distribution of the classical trajectories inside the ferromagnet (Sec. II). The length distribution depends on the geometrical properties of the attached ferromagnet and the connecting boundaries. In Sec. III we specify the classical length distribution for our particular case of the F-film geometry depicted in Fig. 1. We assume that the boundaries of the F film are disordered, leading to complete diffusive reflection of the quasiparticles from these boundaries. We also take into account band mismatch and disorder at the FS interface, which leads to an enhanced backscattering from this interface. For simplicity, we assume a single value of the FS-interface transparency $T$. With the calculated distribution, the DOS at all energies is obtained as a function of the superconducting gap $\Delta$; exchange field $h$; thickness of the $\mathrm{F}$ layer, $d$; and transparency $T$.

We analyze the DOS for different regimes of $h / \Delta$. It shows the interplay between ferromagnetism and superconductivity depending qualitatively on the thickness $d$. For example, a weak exchange field leads to a spin splitting of the DOS, which results in a distinctive low-energy peak in the total DOS. In addition there is an overall suppression of the superconducting features of the DOS with increasing $h$. At higher exchange fields, the DOS shows as a signature of the exchange splitting an oscillatory behavior as a function of the layer thickness. This oscillation of DOS was observed in the experiments. ${ }^{14}$ Our findings are summarized in the following list.

(i) $h=0$ (Sec. IV A). Andreev levels are governed by the distribution of trajectory lengths, which only depends on the geometric properties of the sample. At small energies $E$ $\ll v_{\mathrm{F}} / d$ the DOS is strongly suppressed, originating from the exponential suppression of long trajectories. The DOS at larger energies reflects the length distribution. In our model it display a multiple-peak structure, resulting from multiple reflections at the NS interface. The resulting minigap corresponds to the gap found in a calculation including impurity scattering. ${ }^{25}$

(ii) $h / \Delta<1$ (Sec. IV B). A small exchange field "splits" the DOS for spin-up and- down quasiparticles; i.e., the total DOS is more or less a superposition of "normal" DOS at energies $E \pm h$. Accordingly, the former minigap in the DOS is destroyed. It only remains a dip in the DOS shifted to finite energies. The density of states at the Fermi level approaches the normal-state values in an oscillatory way, i.e., overshooting the normal DOS for certain values of $h$.

(iii) $h / \Delta \geqq 1$ (Sec. IV C). The superconducting features of the DOS are stronger suppressed. The former peaks at $\pm \Delta$ are inverted into dips for thicker layers. Above the gap peaks at $E= \pm h$ appear as the signature of resonant transmission through the ferromagnetic film. For thin layers features at $\pm h$ are absent and the DOS approaches a BCS form.

(iv) $h / \Delta \gg 1$ (Sec. IV D). For layers with $d \gtrsim v_{\mathrm{F}} / h$ the DOS exhibits coherent oscillation, i.e., the form of the DOS difference from the normal-state value becomes independent of $d$. The amplitude and sign, however, depend on the thickness. Only for very low thicknesses $d \ll T v_{\mathrm{F}} / h$ does the DOS approach the BCS form.

In Sec. IV E we condense our results into a map of the proximity DOS. Finally we present some conclusions in Sec. V.

\section{MODEL AND BASIC EQUATIONS}

The system we study is sketched in Fig. 1. A ferromagnetic layer (F) of thickness $d$ is connected to a superconducting bank ( $\mathrm{S}$ ) on one side and bound on the other side by an insulator or vacuum. F is characterized by an exchange splitting, which we take into account as mean field $h$ in the Hamiltonian. The thickness $d$ is larger than the Fermi wave length $\lambda_{\mathrm{F}}$ and smaller than the elastic mean free path $l_{\text {imp }}$, which allows for a quasiclassical description ${ }^{26}$ in the clean limit. We apply the Eilenberger equation in the clean limit:

$$
\begin{aligned}
-i \mathbf{v}_{\mathrm{F}} \boldsymbol{\nabla} \hat{g}_{\sigma}\left(E, \mathbf{v}_{\mathrm{F}}, \mathbf{r}\right)=\left\{[E+\sigma h(\mathbf{r})] \hat{\tau}_{3}\right. \\
\left.-i \hat{\tau}_{2} \Delta(\mathbf{r}), \hat{g}_{\sigma}\left(E, \mathbf{v}_{\mathrm{F}}, \mathbf{r}\right)\right\} .
\end{aligned}
$$

The matrix Green's function for spin $\sigma$ has the form

$$
\hat{g}_{\sigma}=\left(\begin{array}{cc}
g_{\sigma} & f_{\sigma} \\
f_{\sigma}^{\dagger} & -g_{\sigma}
\end{array}\right) .
$$

It depends on energy $E$, the direction of the Fermi velocity $\mathbf{v}_{\mathrm{F}}$, and the coordinate $\mathbf{r}$. Here $\hat{\tau}_{i}$ denote the Pauli matrices, $\Delta(\mathbf{r})$ is the superconducting pair potential (taken as real), and $\sigma(= \pm 1)$ labels the electron spin. The matrix Green's functions obey the normalization condition $\hat{g}_{\sigma}^{2}=1$. Inside the F layer $h$ is constant and $\Delta=0$. We neglect a depression of the pair potential close to the FS interface; thus, $\Delta(\mathbf{r})$ $=$ const inside the superconductor, which applies in the case of a bad contact between the ferromagnet and the superconductor. Strictly speaking, we would have to include an elastic 
collision term in Eq. (1), even in the limit $l_{\text {imp }} \gg d$. However, changes in the spectrum due to this term are limited to small energies $\lesssim v_{\mathrm{F}} / l_{\text {imp }} \ll \min \left(v_{\mathrm{F}} / d, h\right),{ }^{25}$ which are negligible in all cases we study, except for the case $h=0$. Disorder in the superconductor can be neglected in the limit of small interface transmission, which we mostly assume.

We have to solve Eq. (1) along each classical trajectory with length $l$ in $\mathrm{F}$, which comes from the superconductor and ends there. As boundary conditions the solutions approach the bulk values of $\hat{g}_{\sigma}$ at the beginning and the end of a trajectory deep inside the superconductor. These are given by $\hat{g}_{\sigma}($ bulk $)=\left(-i E \hat{\tau}_{3}+\Delta \hat{\tau}_{1}\right) / \sqrt{\Delta^{2}-E^{2}} \cdot{ }^{27}$ It turns out that on a trajectory inside $\mathrm{F}$ the normal Green function $g_{\sigma}$ is constant. It depends only on the length of that trajectory $l$ and is given by

$$
g_{\sigma}=\tanh \left[(-i E-i \sigma h) l / v_{\mathrm{F}}+\arcsin (-i E / \Delta)\right] .
$$

To find the density of states per trajectory, we have to calculate

$$
N(E, l)=\frac{N_{0}}{2} \sum_{\sigma= \pm 1} \operatorname{Re} g_{\sigma}\left(E+i 0, \mathbf{v}_{\mathrm{F}}, \mathbf{r}\right)
$$

As a result we obtain for energies below the gap $(|E| \leqslant \Delta)$

$$
N(E, l)=\frac{N_{0}}{2} \sum_{\sigma= \pm 1} \frac{\pi v_{\mathrm{F}}}{|E+\sigma h|} \sum_{n=-\infty}^{\infty} \delta\left(l-l_{n}\right)
$$

where

$$
l_{n}=\frac{v_{\mathrm{F}}}{E+\sigma h}[n \pi+\arccos (E / \Delta)]
$$

Above the gap $(|E|>\Delta)$ we find

$$
N(E, l)=\frac{N_{0}}{2} \sum_{\sigma= \pm 1} \sum_{n=-\infty}^{\infty} \frac{\operatorname{acosh}|E / \Delta|}{\left[(E+\sigma h) l / v_{\mathrm{F}}-n \pi\right]^{2}+(\operatorname{acosh}|E / \Delta|)^{2}} .
$$

Here $N_{0}$ is the density of states at the Fermi level in the normal state. Equation (5) expresses the fact that the density of states below $\Delta$ is a sum of $\delta$ peaks resulting from Andreev bound states of electrons of $E \geqslant 0$ (positive $n$ 's) and holes of $E<0$ (negative $n$ 's). The energies also follow from the quasiclassical quantization condition $l=l_{n}$.

The total DOS is obtained by averaging the expressions (5) and (7) over all classical trajectories. Denoting the trajectory length distribution by $p(l)$ and using Eq. (5), we find for the subgap DOS

$$
N(E)=\int d l p(l) N(E, l)=\frac{N_{0}}{2} \sum_{\sigma= \pm 1} \frac{\pi v_{\mathrm{F}}}{|E+\sigma h|} \sum_{n=-\infty}^{\infty} p\left(l_{n}\right) \quad \text { for } \quad|E| \leqslant \Delta .
$$

This formula is a general result for the subgap density of states of a quasiballistic metal connected to a superconductor. It is completely specified by the length distribution of classical trajectories, which depends only on the geometrical properties of the attached ferromagnet and the surrounding boundaries.

Averaging expression (7) over $p(l)$ the total DOS for energies above the gap is

$$
N(E)=\frac{N_{0}}{2} \sum_{\sigma= \pm 1} \sum_{n=-\infty}^{\infty} \int d l p(l) \frac{\operatorname{acosh}|E / \Delta|}{\left[(E+\sigma h) l / v_{\mathrm{F}}-n \pi\right]^{2}+(\operatorname{acosh}|E / \Delta|)^{2}} \text { for }|E|>\Delta .
$$

The absence of discrete bound states reflects the fact that the Andreev reflection at energies above the gap is incomplete. Therefore, the quasiparticle states in the ferromagnet are determined by "scattering resonances" of quasiparticles incident from the superconductor.

\section{DISTRIBUTION OF THE TRAJECTORY LENGTH}

Now we specify the length distribution for our particular case. We model the F layer by a weakly disordered thin film bounded by a rough surface to the insulator and a rough FS interface of average transparency $T$. A typical classical trajectory is depicted in Fig. 1. An electron coming from the bulk of $\mathrm{S}$ enters into the $\mathrm{F}$ layer and after several reflection from the insulator and the FS interface returns to the $S$ bank, where it is Andreev reflected as a hole and transverses the trajectory in the opposite direction. Thus, the building block of a trajectory is the segment between two successive reflec- tions from S. The number of blocks which form the total trajectory depends on the transparency of the interface; i.e., it is roughly $\sim 1 / T$.

As a first step, we consider the length distribution in the case of a perfectly transparent FS interfaces, in which the length distribution is that of one elementary block. Due to the roughness of the insulator and the FS interface, the quasiparticles undergo diffusive reflection from these boundaries. Incident and reflected directions are completely uncorrelated. Then, assuming an uniform distribution for the directions of $\mathbf{v}_{\mathrm{F}}$, we obtain for the length distribution of one elementary block (corresponding to the case of $T=1$ ) 


$$
p_{0}(l)=\int_{0}^{1} d\left(\cos \theta_{i}\right) \int_{0}^{1} d\left(\cos \theta_{r}\right) \delta\left(l-\frac{d}{\cos \theta_{i}}-\frac{d}{\cos \theta_{r}}\right),
$$

where $\theta_{i}\left(\theta_{r}\right)$ denotes angle of the incident (reflected) direction with respect to the normal to surface of the insulator. To take into account the weak bulk disorder, we include a factor exp $-l / l_{\text {imp }}$. This serves mainly to yield a finite average length of the distribution (10). In a purely ballistic layer with $p_{0}(l)$ given by Eq. (10), the average length would logarithmically divergent. Taking this into account we obtain

$$
p_{0}(l)=\frac{2 d}{C l^{2}}\left[\frac{l-2 d}{l-d}+\frac{2 d}{l} \ln \frac{l-d}{d}\right] e^{-l / l_{\mathrm{imp}} \theta}\left(\frac{l}{d}-2\right),
$$

where $C=E_{2}^{2}\left(d / l_{\text {imp }}\right)\left[E_{2}(z)=\int_{1}^{\infty} d x \exp (-z x) / x^{2}\right.$ is the exponential integral of order 2].

In the second step, we connect the elementary building blocks if the FS interface has a transparency $T<1$. In determining the length distribution we assume that a particle either goes through the interface or is fully reflected. Only the number of these reflection depends on $T$. We do not take into account quantum mechanical interference for a single reflection at the FS interface. Taking this into account will lead essentially to the same results as our approach. By an expansion in the reflectivity $R=1-T$ for the distribution $p(l)$ we can write

$$
p(l)=T \sum_{n=0}^{\infty} R^{n} \int d l_{0} \cdots d l_{n} p_{0}\left(l_{0}\right) \cdots p_{0}\left(l_{n}\right) \delta\left[l-\sum_{i=0}^{n} l_{i}\right],
$$

where the $n$th term in the expansion is the contribution of the trajectories on which quasiparticles after $n$ times reflections from the FS interface leave the F layer. It is easy to see from Eq. (12) that $p(l)$ obeys the integral equation

$$
p(l)=T p_{0}(l)+R \int d l^{\prime} p_{0}\left(l^{\prime}\right) p\left(l-l^{\prime}\right),
$$

which is readily solved by a Fourier transformation:

$$
p(l)=\int_{-\infty}^{\infty} \frac{d k}{2 \pi} e^{i k l} P(k) .
$$

Replacing Eq. (14) in Eq. (13) we find

$$
P(k)=\frac{T P_{0}(k)}{1-R P_{0}(k)},
$$

where $P_{0}(k)=E_{2}^{2}\left(i k d+d / l_{\text {imp }}\right) / C$ is the Fourier transform of $p_{0}(l)$.

The distribution $p(l)$ determines the relevant length scale associated with the geometrical size of the system corresponding to the typical distances quasiparticles travel inside F. We have plotted $p(l)$ for different $T$ 's and $d / l_{\text {imp }}=0.1$ in Fig. 2. For small $T$, it has a characteristic double-peak structure close to the shortest trajectories $l \simeq 2 d$, resulting from trajectories reflected once and twice from the insulator. At

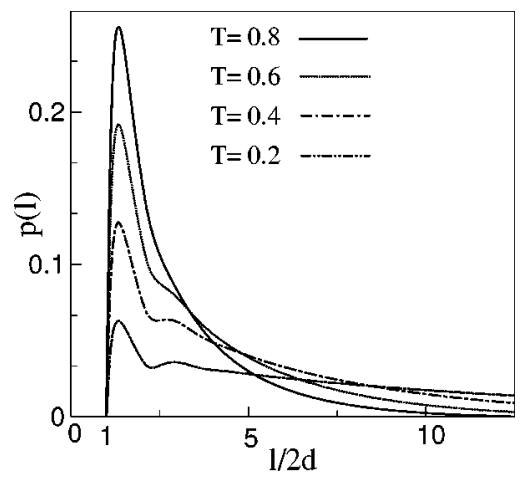

FIG. 2. The calculated distribution of the trajectory length in the F layer for different values of FS transparency. For small FS transparency the double-peak structure close to the smallest length originates from the first two reflections, whereas the distribution for long trajectories decays as $\exp (-l / \bar{l})$, with the average length $\bar{l} \approx 2 d / T$.

large $l$ the distribution decays exponentially as $\exp (-l / \bar{l})$, where $\bar{l} \simeq 2 d \ln \left(l_{\mathrm{imp}} / d\right) / T$ is the mean trajectory length. We therefore have two characteristic lengths of the distribution: the smallest possible trajectory length $2 d$ and the average length $\bar{l}$. The former determines the energy of the first Andreev level and the latter the possible longest length of the trajectories. For $T \sim 1$ these two length scale are of the same order, leaving the thickness as the only relevant length scale. In this case $p(l)$ has only one peak close to $2 d$ (see Fig. 2). Which of two length scales $2 d$ and $\bar{l}$ determines the total density of states will depend on the other parameters.

Combining Eqs. (8) and (14) we obtain for the total subgap DOS

$$
N(E)=\frac{N_{0}}{2} \sum_{\sigma= \pm} \sum_{n=-\infty}^{\infty} P\left(k_{n}\right) e^{2 n i \arccos E / \Delta},
$$

where $k_{n}=2 n(E+\sigma h) / v_{\mathrm{F}}$. Similarly, from Eqs. (9) and (14) the total DOS for the energies above the gap is obtained:

$$
N(E)=\frac{N_{0}}{2} \sum_{\sigma= \pm} \sum_{n=-\infty}^{\infty} P\left(k_{n}\right) e^{-2|n| \operatorname{acosh}|E / \Delta|} .
$$

Thus, in both cases the density of states is fully expressed in terms of the Fourier transform of the trajectory length distribution. Most probably a real $\mathrm{F}$ film has a nonuniform thickness due to the large scale roughness of the boundaries. Assuming a smoothly varying thickness we can take this into account by averaging expressions (16) and (17) over a Gaussian distribution of the thickness around a mean value $d$. This will also leads to a smoothening of the sharp features in DOS resulting from the lower cutoff in $p(l)$. The qualitative behavior will, however, not change. In our calculations, we have taken a width of the distribution to be of order $10 \%$ that correspond to condition of the experiments. ${ }^{14}$

\section{RESULTS AND DISCUSSIONS}

Equations (16) and (17) express the DOS of a $F$ layer contacted by the superconductor in terms of the trajectory 

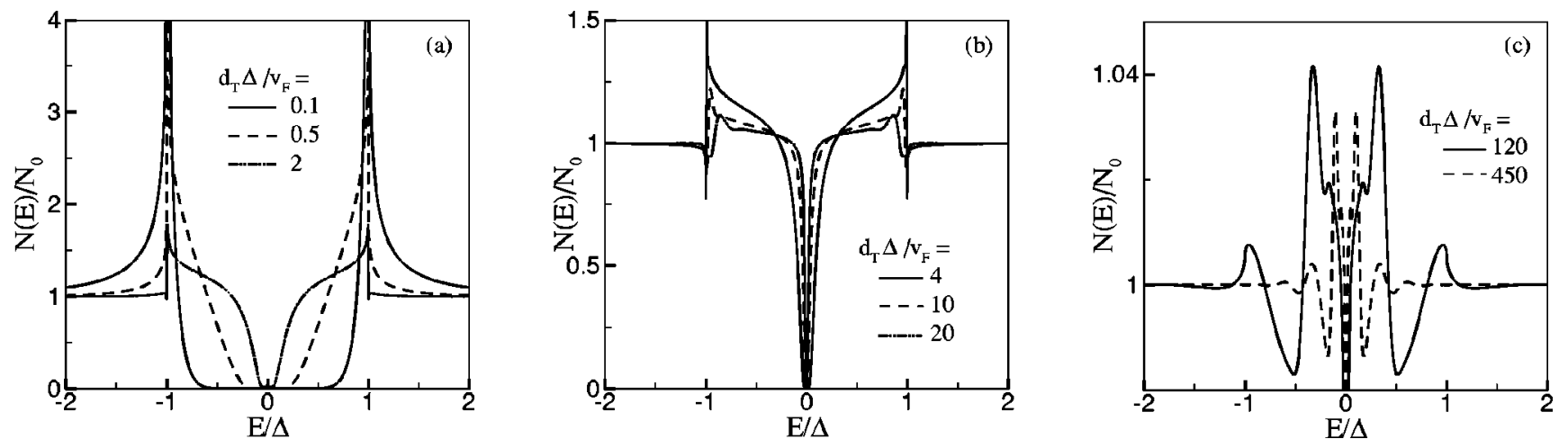

FIG. 3. The DOS vs energy of the normal layer $(h=0)$ for different values of $d_{T} \Delta / v_{\mathrm{F}}$. (a) Suppression of the superconducting features of DOS with increasing $d_{T} \Delta / v_{\mathrm{F}}$ and appearance of a minigap of order $v_{\mathrm{F}} / d_{T}$. (b) The first Andreev peaks (AP's) at roughly $\pm v_{\mathrm{F}} / 2 d$ correspond to the first peak in the distribution of the trajectory length. (c) For very large $d_{T} \Delta / v_{\mathrm{F}}$ the DOS has many AP's, leading to small deviations of order $T$ from the normal-state DOS.

length distribution. Depending on the relative values of $\Delta, h$, the Thouless energy $v_{\mathrm{F}} / 2 d$, and $T$, the resulting DOS has different behaviors. We will concentrate mainly on the limit $T \ll 1$. For the length dependence it is useful to distinguish between normal metal with $h=0$, a weak ferromagnet $h$ $\sim \Delta$ of $h<\Delta$ and $h>\Delta$, and a strong ferromagnetic film of $h \gg \Delta$. We analyze the DOS in each case for different values of $d_{T} \Delta / v_{\mathrm{F}}\left(2 d_{T}=\bar{l}\right)$, being the relevant length scale in the limit $T \ll 1$. In the end, we summarize all results in a map of the DOS depending on $d_{T} \Delta / v_{\mathrm{F}}$ and $d_{T} h / v_{\mathrm{F}}$.

\section{A. Normal film}

Let us start with a normal metal film $(h=0)$ contacted by the superconductor. The DOS is shown in Fig. 3, for different values of $d_{T} \Delta / v_{\mathrm{F}}$. In the limit of a very thin layer with $d_{T} \Delta / v_{\mathrm{F}} \ll 1$, the DOS has essentially the form of superconducting DOS with sharp peaks at $E= \pm \Delta$ and zero DOS for energies inside the gap. By increasing $d_{T} \Delta / v_{\mathrm{F}}$ the peaks are getting broader and a finite DOS appears at small energies. There is still an energy interval around $E=0$ with zero DOS [see Fig. 3(a)]. Increasing $d_{T} \Delta / T$ further leads to a suppression of the superconducting features of the DOS. The zero DOS interval become smaller and the DOS at other energies tends to be closer to the DOS of the normal state. Thus, the density of states develops a minigap around the Fermi level, which decreases with increasing $d_{T}$ roughly as $v_{\mathrm{F}} / d_{T}$ $=v_{\mathrm{F}} T / d \ln \left(l_{\mathrm{imp}} / d\right)$ [see Fig. 3(b)]. This minigap is related to the mean length of the trajectories $\bar{l}$, which has a finite value, if $d / l_{\text {imp }}$ is finite. The presence of weak bulk disorder in the normal film suppresses long trajectories. Formally, this was included in the distribution of the trajectory length as the exponentially decaying factor in Eq. (11), which leads to the finite mean length $\bar{l}=2 d \ln l_{\text {imp }} / d$. This acts as an effective upper limit of order $\bar{l}$ of the length of the trajectories, which gives a lower bound to the energy of the Andreev bound states. Similar features were found before within a tunneling model $^{2}$ and in the diffusive models ${ }^{28,29}$ in a disordered normal layer contacted by the superconductor.

The peaks of $E= \pm \Delta$ originate from the first peak in the distribution $p(l)$ at $l \simeq 2 d$, which at higher $d_{T} \Delta / v_{\mathrm{F}}$ move to lower energies given roughly by $\pm v_{\mathrm{F}} / 2 d$ [see Fig. 3(b)]. They originate from Andreev peaks (AP's) resulting from trajectories with $l \simeq 2 d$. By increasing $d_{T} \Delta / v_{\mathrm{F}}$ the first AP moves to lower energies, and when $d_{T} \Delta / v_{\mathrm{F}} \gtrsim 1 / T$, the next $\mathrm{AP}$ appear at $E= \pm \Delta$ [Fig. 3(c)]. In this case the DOS is close to the normal-state values. Small deviations proportional to $T$ display many AP's, as shown in Fig. 3(c). Small peaks close the main peaks, which are more pronounced for the first AP, correspond to the second peak of $p(l)$ at $l$ $\simeq 4 d$.

\section{B. Very weak ferromagnet, $h<\Delta$}

Now we study the effect of the spin splitting in a ferromagnetic film on the DOS. First we consider the case of a weak ferromagnet, where the exchange field is of order of the superconducting gap, but $h<\Delta$. A qualitative picture of the influence of $h$ on the DOS follows from the condition (6) for the formation of Andreev bound states in the ferromagnetic layer. This is the semiclassical quantization condition for coherent superposition of two subsequent Andreev reflection of a quasiparticle from $\mathrm{S}$, which propagate along a trajectory of length $l$. It includes the phase gained by a quasiparticle of spin $\sigma$ along a trajectory, $(E+\sigma h) l$, and the phase shift produced by an Andreev reflection, $\arccos (E / \Delta)$. If the energy of the quasiparticle is not close to $\Delta$, the phase shift resulting from Andreev reflection is different from zero. To obtain constructive interference the total phase must be an integer multiple of $2 \pi$, as follows from Eq. (6). The existence of an upper limit on the length of the trajectories (as discussed above) leads to the formation of a zero DOS interval (minigap) around $E= \pm h$. Therefore, the total subgap DOS should be similar to the average of two by $\pm h$ shifted normal spectra.

In column (I) of Fig. 4 the DOS of the ferromagnetic film with $h=0.5 \Delta$ is shown for different values of $d_{T} \Delta / v_{\mathrm{F}}$. Shifting the minigap leads to minima at $E= \pm h$. The zeroenergy DOS becomes finite and increases with increasing $d_{T} \Delta / v_{\mathrm{F}}$. At higher $d_{T} \Delta / v_{\mathrm{F}} \sim 1$ the DOS has a smooth peak at zero energy and two dips at $E= \pm h$ [see Fig. 4(Ia)]. Here, the AP are located at $E= \pm \Delta$. By increasing $d_{T} \Delta / v_{\mathrm{F}}$ the width of the dips decreases roughly as $v_{\mathrm{F}} / d_{T}$ and the first AP 
$(I) \frac{h}{\Delta}=0.5$
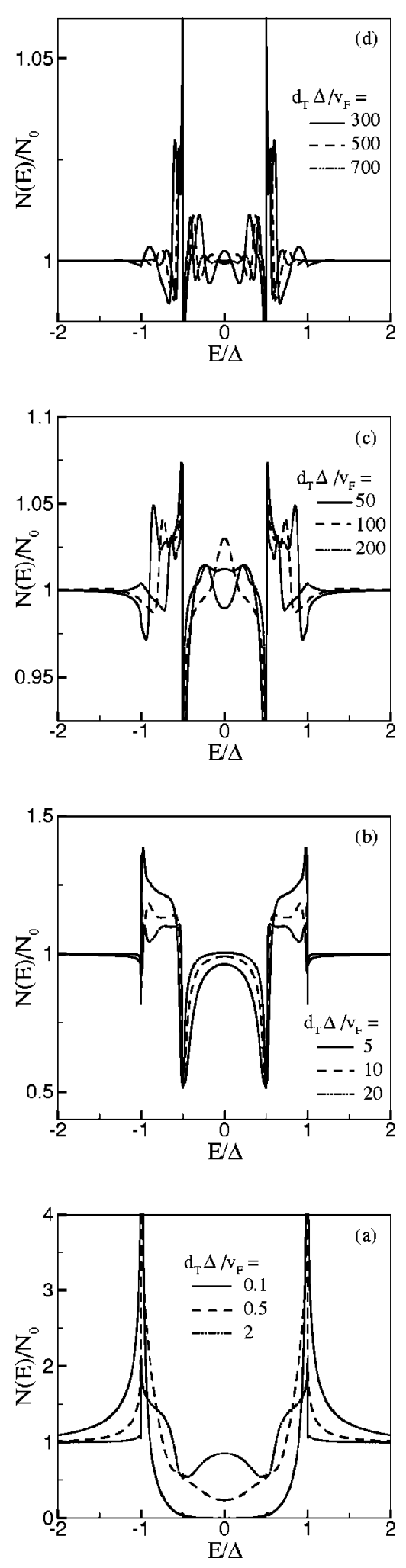

$(I I) \frac{h}{\Delta}=1.5$
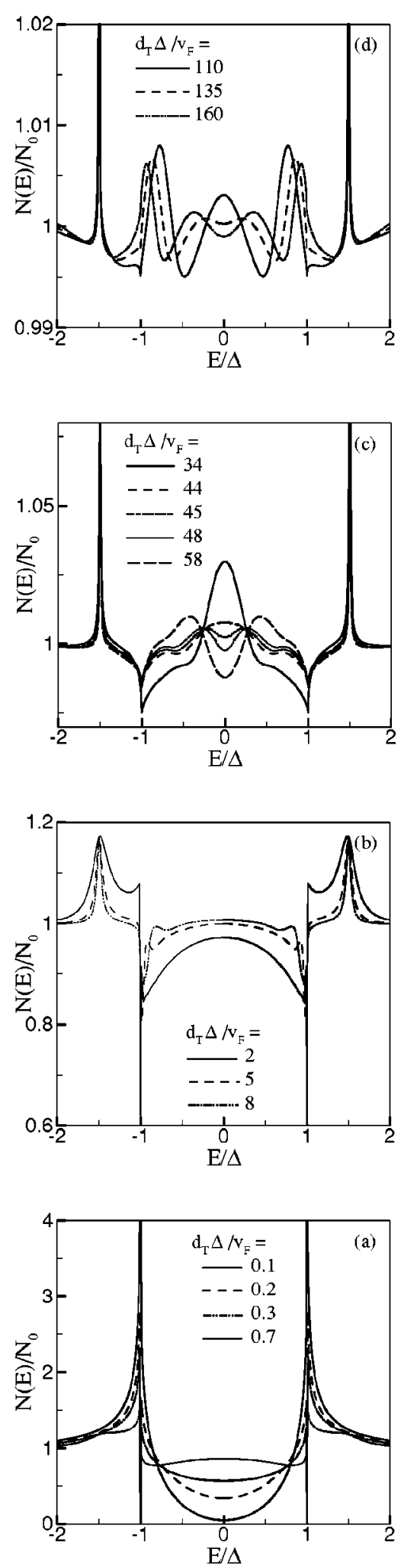

$(I I I) \frac{h}{\Delta}=10$
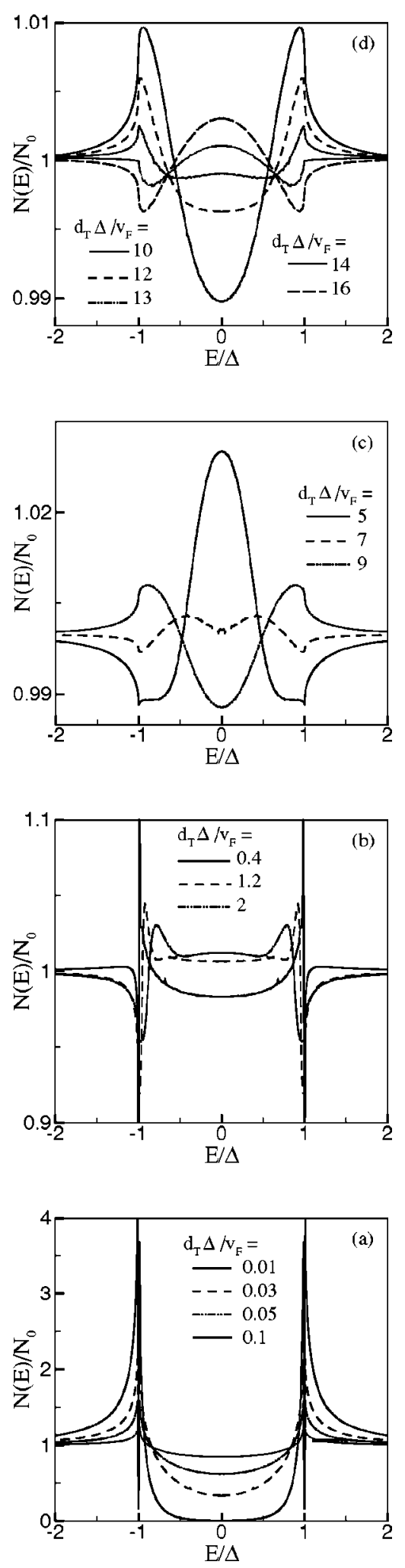

FIG. 4. Density of states in a ferromagnetic layer in contact with a superconductor. Different columns labeled (I)-(III) correspond to the values of the exchange field given above. The thickness of the layer is increased from the bottom to the top. For an explanation of the various regimes, see the text. 
moves to energies below the gap, i.e., $\sim \pm v_{\mathrm{F}} / d$ [see Fig. $4(\mathrm{Ib})]$. We can distinguish two domains of energies below the exchange field $|E|<h$ and energies above the exchange field $|E|>h$. At higher $d_{T} \Delta / v_{\mathrm{F}}$ the first AP moves from $|E|>h$ to $|E|<h$ and the next AP appears at $|E|>h$. For the region $|E|<h$ the DOS shows a zero energy peak, if the first APs merge at $E=0$ [see Fig. 4(Ic)]. This is a zero-energy Andreev peak (ZEAP), which originates from phase shifting caused by the exchange field. Additional shifting of the AP results in an oscillatory behavior of the DOS in the domain $|E|<h$. As shown in Fig. 4(Id), in the limiting case of $d_{T} \Delta / v_{\mathrm{F}} \gg 1 / T$ the DOS is close to the normal-state value, exhibiting small deviations, which are of the order of the FS-interface transparency $T$. The deviations have the form of small oscillations at all energies.

\section{Weak ferromagnet, $h>\Delta$}

In the case of $h>\Delta$ the suppression of the superconducting features of DOS occurs at lower $d_{T} \Delta / v_{\mathrm{F}}$, compared with the previous case of $h<\Delta$ [see Fig. 4(IIa)]. The subgap DOS has similar features as it had for energies below $h$ in the case of $h<\Delta$. The zero-energy DOS increases to the normal-state values as $d_{T} h / v_{\mathrm{F}}$ becomes of order unity. Then, the DOS has a smooth peak at $E=0$ and minima at $E= \pm \Delta$, as is shown in Figs. 4(IIb) and 4(IIc). The AP at $E= \pm \Delta$ move to lower energies at a higher values of $d_{T} \Delta / v_{\mathrm{F}}$ and form ZEAP, when they merge at $E=0$ [see Fig. 4(IIc)]. The size of the ZEAP is of order of $T$.

The main feature of DOS in energies above the gap consists of sharp peaks at $E= \pm h$ [see Figs. 4(IIb)-4(IId)]. These peaks originate from a resonant transmission of the quasiparticles through the superconducting potential ( $\Delta$ $=0$ ) inside the F film. A quasiparticle incident from the superconductor to the $\mathrm{F}$ film with energy above the gap is scattered by the potential well whose width is determined by the length of the quasiparticle trajectory $l$ inside F. The phase gained by the quasiparticle of spin $\sigma$ inside the potential well is given by $(E+\sigma h) l$. At $E=-\sigma h$ the incident and transmitted quasiparticles interfere constructively, which leads to a reflectionless transmission. Similar effect were found before in normal-metal-superconductor hetrostructures. ${ }^{30}$

For $d_{T} h / v_{\mathrm{F}}>1 / T$ the subgap DOS shows an oscillatory behavior around the normal state value as a function of $d h / v_{\mathrm{F}}$ [see Figs. 4(IIc) and 4(IId)]. The period of the oscillation is $\pi / 2$ and the amplitude is of order $T$. The amplitude is damped in the limit of $d_{T} \Delta / v_{\mathrm{F}} \gg 1 / T$. Note that the phase of the oscillation depends on the energy.

\section{Ferromagnetic film}

Now let us consider rather strong exchange fields $h \gg \Delta$. In column III of Fig. 4 we plotted $N(E)$ for $h=10 \Delta$ at different $d_{T} \Delta / v_{\mathrm{F}}$. As shown in Fig. 4(IIIa) the suppression of the superconducting features from DOS by increasing $d_{T} \Delta / v_{\mathrm{F}}$ is faster than in the weak-ferromagnetic cases. In fact the DOS reaches almost to the normal-state value for $d_{T} h / v_{\mathrm{F}} \sim 1$. As long as $d_{T} h / v_{\mathrm{F}} \lesssim 1$, mainly long trajectories of $l \sim \bar{l}$ contribute to the zero-energy DOS, which are well approximated by an exponential distribution of the form $\exp (-l / \bar{l})$. Replacing this approximation form of $p(l)$ into the general expression of the DOS, Eq. (8), we find that the zero-energy DOS increases with $d_{T} h / v_{\mathrm{F}}$ roughly as

$$
N(0)=N_{0} \frac{\pi v_{\mathrm{F}}}{h \bar{l}} \frac{\exp \left(-\pi v_{\mathrm{F}} / 2 h \bar{l}\right)}{1-\exp \left(-\pi v_{\mathrm{F}} / h \bar{l}\right)} .
$$

This result is also applicable for the weak-ferromagnetic case discussed above. As before, the shifting of the AP to lower energies [see Fig. 4(IIIb)] leads to the formation of a ZEAP at the Fermi level as is seen from Figs. 4(IIIc) and 4(IIId). Then the DOS develops coherent oscillation as a function of $d h / v_{\mathrm{F}}$ with period $\pi / 2$. The amplitude and the sign of the oscillation depend on energy. Maximal amplitudes of opposite sign always occur at zero energy or at the gap energy [see Fig. 4(IIId)]. This results in an inverted energy dependence of the DOS by changing $d$, which has been observed in the experiment. ${ }^{14}$ We have shown in Ref. 24 that our results is in a quantitative agreement with the experimental data.

\section{E. Maps of the proximity DOS}

Summarizing the above analysis we present a map showing the dependence of DOS on $d_{T} \Delta / v_{\mathrm{F}}$ and $d_{T} h / v_{\mathrm{F}}$ for small FS-interface transparency. This map is shown in Fig. 5. Various regions in the map are distinguished by different ranges of $h / \Delta$ and $d_{T} \Delta / v_{\mathrm{F}}$ (or equivalently $d_{T} h / v_{\mathrm{F}}$ ). Along the diagonal lines $h$ is equal to $\Delta$ and moving upwards $d_{T} \Delta / v_{\mathrm{F}}$ (and consequently $d_{T} h / v_{\mathrm{F}}$ ) increases. The quarter circles are curves with constant $d_{T}$, along which the ratio $h / \Delta$ is varying. In the following we discuss different regions according to this classification. The normal film corresponds to the vertical axis $(h=0)$, which consists of three parts. The first part is limited by $d_{T} \Delta / v_{\mathrm{F}} \lesssim 1$. Here the superconducting features are dominant at lower $d_{T} \Delta / v_{\mathrm{F}}$ and suppressed for $d_{T} \Delta / v_{\mathrm{F}}$ $\sim 1$, showing a minigap at the Fermi level. The second part is limited by $1 \lesssim d_{T} \Delta / v_{\mathrm{F}} \lesssim 1 / T$, where the main feature is the first AP at energies $\sim \pm v_{\mathrm{F}} / 2 d$ and a minigap of order $v_{\mathrm{F}} / d_{T}$. Close to the boundary $d_{T} \Delta / v_{\mathrm{F}} \sim 1 / T$ the second AP appears in the DOS. Finally, the third part is the region $d_{T} \Delta / v_{\mathrm{F}}$ $>1 / T$, where the DOS contains many AP, appearing as small deviations (proportional to $T$ ) from the normal state DOS.

In the case of a ferromagnetic film we distinguish the following regions:

(i) The strongly superconducting region is limited by the smallest quarter circle in which the superconducting features are dominate the DOS. At nonzero $h$ the zero-energy DOS appears at larger $d_{T} \Delta / v_{\mathrm{F}}$ and the DOS increases to the value of the normal state in the domain close to the second-quarter circle boundary. For the part $h<\Delta$, there is a smooth maximum at $E=0$ between two minima at $E= \pm h$. For $h>\Delta$ we have only a smooth peak at $E=0$.

(ii) The intermediate regions limited by two quarter circles. In $h<\Delta$ part the main feature in DOS is the existence of two dips at $E= \pm h$ and the first AP. Close to the second boundary we observe a separation between two energy domains $|E|<h$ and $|E|>h$. While the second AP peaks 


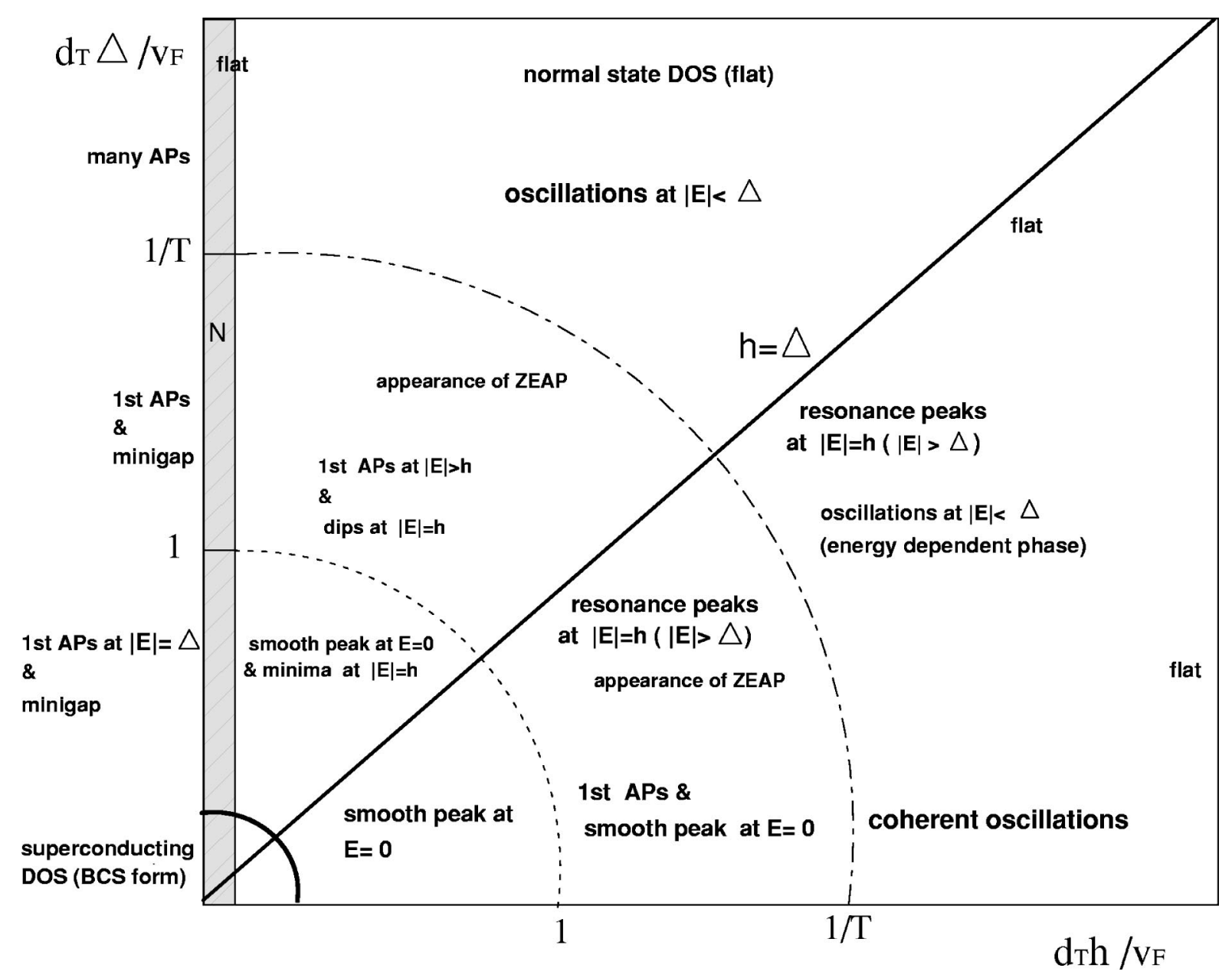

FIG. 5. Map of the proximity DOS in a ferromagnetic layer showing the dependence on $d_{T} \Delta / v_{\mathrm{F}}$ and $d_{T} h / v_{\mathrm{F}}$ for a small FS-interface transparency $T \ll 1$. In the case of high transparency $T \sim 1$ the region between 1 and $1 / T$ is absent.

appears at energies above $h$, the first AP peaks move to energies below $h$ domain. In the $h>\Delta$ part the DOS has a smooth peak at $E=0$. In the domain close to the diagonal line $h \geqslant \Delta$, there are also two resonance sharp peaks at $E=$ $\pm h$ which disappear in $h \gg \Delta$ regions. In both cases of $h$ $<\Delta$ and $h>\Delta$, shifting of the AP's leads to the formation of a ZEAP. This happens at regions close to the third quarter circle.

(iii) In the region above the third-quarter circle the DOS is close to the normal-state value (flat). There are, however, small deviations proportional to $T$, which have different origins in the different domains. In the limiting domains of $h$ $\ll \Delta$ and $h \gg \Delta$ (close to the respective axis) they consist of many AP's and oscillations, respectively. In the $h<\Delta$ part there are many AP's above $h$ and an oscillatory variation below $h$. In the $h>\Delta$ part and for weak exchange fields in the subgap part we have oscillatory variations with an energy-dependent sign and amplitude, which result from a collective shift of many AP's. As the intermediate region the resonance peaks are present at $E= \pm h$. In the strongexchange field part these peaks are disappear. Here the oscillations of subgap DOS are produced by the first AP only.

The DOS map for the case of high transparency $T \sim 1$ is similar to Fig. 5. The difference is the absence of the region between 1 and 1/T on both axis. In the remaining regions we have features similar to the $T \ll 1$ case. The value of the minigap and the energy of first AP are the same order. All features and variations including $\mathrm{AP}$ and oscillations are more pronounced than the $T \ll 1$ case.

\section{CONCLUSION}

In summary, we have studied theoretically superconducting proximity effect in ballistic ferromagnetic layers. Within the quasiclassical formalism, we have obtained expressions for the density of states at all energies in a ferromagnetic metal in contact with a superconductor which are completely specified by Fourier transform of the length distribution of classical trajectories in the ferromagnet. The length distribution of trajectories depends only on the geometrical properties of the attached ferromagnet and the connected interfaces. Thus, the obtained expressions are applicable for ballistic FS structures of arbitrary geometry. We have calculated the length distribution for the film geometry in a quasiballistic model taking into account the finite transparency of the FS interface, the roughness at the film boundaries, and weak bulk disorders. The density of states exhibits a variety of structures depending on the values of the superconducting 
energy gap $\Delta$; the ferromagnet exchange field $h$; the thickness of the F film, $d$; and the FS interface transparency $T$. We have observed many interesting features, like the splitting of the subgap density of states for spin-up and spin-down quasiparticles, a zero-energy Andreev peak, resonant transmission peaks above the gap at $E= \pm h$, and oscillations of the DOS as a function of the film thickness. These effects have been explained in terms of the phase shift of Andreev bound states, caused by the exchange field. We have analyzed the density of states in the full parameter range and summarized the results in a map, shown in Fig. 5.

\section{ACKNOWLEDGMENTS}

One of the authors (M.Z.) would like to thank the group of Professor G.E.W. Bauer at Delft University of Technology for their hospitality and support during his visit.
${ }^{1}$ W. L. McMillan and J. R. Rowell, in Superconductivity, edited by R. D. Parks (Dekker, New York, 1969).

${ }^{2}$ W.L. McMillan, Phys. Rev. 175, 537 (1968).

${ }^{3}$ A.F. Andreev, Sov. Phys. JETP 19, 1228 (1964).

${ }^{4}$ S. Guéron, H. Pothier, N.O. Birge, D. Esteve, and M. Devoret, Phys. Rev. Lett. 77, 3025 (1996).

${ }^{5}$ W. Belzig, C. Bruder, and G. Schön, Phys. Rev. B 54, 9443 (1996)

${ }^{6}$ S.H. Tessmer, M.B. Tarlie, D.J. Van Harlingen, D.L. Maslov, and P.M. Goldbart, Phys. Rev. Lett. 77, 924 (1996).

${ }^{7}$ Y. Levi, O. Millo, N.D. Rizzo, D.E. Prober, and L.R. Motowidlo, Phys. Rev. B 58, 15128 (1999).

${ }^{8}$ N. Moussy, H. Courtois, and B. Pannetier, Rev. Sci. Instrum. 72, 128 (2001).

${ }^{9}$ M. Vinet, C. Chapelier, and F. Lefloch, Phys. Rev. B 63, 165420 (2001).

${ }^{10}$ V.I. Fal'ko, C.J. Lambert, and A.F. Volkov, JETP Lett. 69, 532 (1999)

${ }^{11}$ F.J. Jedema, B.J. van Wees, B.H. Hoving, A.T. Filip, and T.M. Klapwijk, Phys. Rev. B 60, 16549 (1999).

${ }^{12}$ W. Belzig, A. Brataas, Yu.V. Nazarov, and G.E.W. Bauer, Phys. Rev. B 62, 9726 (2000).

${ }^{13}$ W.J. Gallagher, D.E. Paraskevopoulos, P.M. Tedrow, S. FrotaPessoa, and B.B. Schwartz, Phys. Rev. B 21, 962 (1980); see also R. Meservey and P.M. Tedrow, Phys. Rep. 238, 173 (1994).

${ }^{14}$ T. Kontos, M. Aprili, J. Lesueur, and X. Grison, Phys. Rev. Lett. 86, 304 (2001).

${ }^{15}$ L.B. Ioffe, V.B. Geshkenbein, M.V. Feigel'man, A.L. Fauchere, and G. Blatter, Nature (London) 398, 679 (1999).

${ }^{16}$ J.E. Mooij, T.P. Orlando, L. Levitov, L. Tain, C.H. van der Wal, and S. Lioyd, Science 285, 1036 (1999).

${ }^{17}$ A.I. Larkin and Yu.N. Ovchinnikov, Sov. Phys. JETP 20, 762 (1995)
${ }^{18}$ P. Fulde and R.A. Ferrel, Phys. Rev. 135, A550 (1964).

${ }^{19}$ Z. Radovic, M. Ledvij, L. Dobrosavljevic-Grujic, A.I. Buzdin, and J.R. Clem, Phys. Rev. B 44, 759 (1991).

${ }^{20}$ J.S. Jiang, D. Davidovic, Daniel H. Reich, and C.L. Chien, Phys. Rev. Lett. 74, 314 (1995); Th. Mühge, N.N. Garif'yanov, Yu.V. Goryunov, G.G. Khaliullin, L.R. Tagirov, K. Westerholt, I.A. Garifullin, and H. Zabel, ibid. 77, 1857 (1996).

${ }^{21}$ J. Aarts et al., Phys. Rev. B 56, 2779 (1997); L. Lazar et al., ibid. 61, 3711 (2000); M. Schöck, C. Sürgers, and H.v. Löhneysen, Eur. Phys. J. B 14, 1 (2000).

${ }^{22}$ V.V. Ryazanov, A.V. Veretennikov, V.A. Oboznov, A.Yu. Rusanov, V.A. Larkin, A.A. Golubov, and J. Aarts, Physica B 284-288, 495 (2000); V.V. Ryazanov, V.A. Obozno, A.Yu. Rusanov, A.V. Veretennikov, A.A. Golubov, and J. Aarts, Phys. Rev. Lett. 86, 2427 (2001).

${ }^{23}$ L.N. Bulaevskii, V.V. Kuzii, and A.A. Sobyanin, Pis'ma Zh. Éksp. Teor. Fiz. 25, 314 (1977) [JETP Lett. 25, 290 (1997)].

${ }^{24}$ M. Zareyan, W. Belzig, and Yu.V. Nazarov, Phys. Rev. Lett. 86, 308 (2001).

${ }^{25}$ S. Pilgram, W. Belzig, and C. Bruder, Phys. Rev. B 62, 12462 (2000).

${ }^{26}$ G. Eilenberger, Z. Phys. 214, 195 (1968); A.I. Larkin and Yu.N. Ovchinnikov, Sov. Phys. JETP 26, 1200 (1968).

${ }^{27}$ I.O. Kulik and A.N. Omelyanchouk, Sov. J. Low Temp. Phys. 4, 142 (1978).

${ }^{28}$ A.A. Golubov and M.Yu. Kupriyanov, J. Low Temp. Phys. 70, 83 (1988); Sov. Phys. JETP 69, 805 (1989).

${ }^{29}$ W. Belzig, Ph.D. thesis, Karlsruhe University, 1999.

${ }^{30}$ A. Kastalsky, A.W. Kleinsasser, L.H. Greene, R. Bhat, and F.P. Milliken, Phys. Rev. Lett. 67, 3026 (1991); C. Nguyen, H. Kroemer, and E.L. Hu, ibid. 69, 2847 (1992); see also M. Schechter, Y. Imry, and Y. Levinson, Phys. Rev. B 64, 224513 (2001). 